\title{
Beam-excited whistler waves at oblique propagation with relation to STEREO radiation belt observations
}

\author{
K. Sauer and R. D. Sydora \\ Department of Physics, University of Alberta, Edmonton, AB, T6G 2G7, Canada \\ Received: 21 February 2010 - Revised: 5 June 2010 - Accepted: 8 June 2010 - Published: 22 June 2010
}

\begin{abstract}
Isotropic electron beams are considered to explain the excitation of whistler waves which have been observed by the STEREO satellite in the Earth's radiation belt. Aside from their large amplitudes $(\sim 240 \mathrm{mV} / \mathrm{m})$, another main signature is the strongly inclined propagation direction relative to the ambient magnetic field. Electron temperature anisotropy with $T_{\mathrm{e} \perp}>T_{\mathrm{e} \|}$, which preferentially generates parallel propagating whistler waves, can be excluded as a free energy source. The instability arises due to the interaction of the Doppler-shifted cyclotron mode $\omega=-\Omega_{\mathrm{e}}+k V_{\mathrm{b}} \cos \theta$ with the whistler mode in the wave number range of $k c / \omega_{\mathrm{e}} \leq$ 1 ( $\theta$ is the propagation angle with respect to the background magnetic field direction, $\omega_{\mathrm{e}}$ is the electron plasma frequency and $\Omega_{\mathrm{e}}$ the electron cyclotron frequency). Fluid and kinetic dispersion analysis have been used to calculate the growth rate of the beam-excited whistlers including the most important parameter dependencies. One is the beam velocity $\left(V_{\mathrm{b}}\right)$ which, for instability, has to be larger than about $2 V_{\mathrm{Ae}}$, where $V_{\mathrm{Ae}}$ is the electron Alfvén speed. With increasing $V_{\mathrm{Ae}}$ the propagation angle $(\theta)$ of the fastest growing whistler waves shifts from $\theta \sim 20^{\circ}$ for $V_{\mathrm{b}}=2 V_{\mathrm{Ae}}$ to $\theta \sim 80^{\circ}$ for $V_{\mathrm{b}}=5 V_{\mathrm{Ae}}$. The growth rate is reduced by finite electron temperatures and disappears if the electron plasma beta $\left(\beta_{\mathrm{e}}\right)$ exceeds $\beta_{\mathrm{e}} \sim 0.2$. In addition, Gendrin modes $\left(k c / \omega_{\mathrm{e}} \approx 1\right)$ are analyzed to determine the conditions under which stationary nonlinear waves (whistler oscillitons) can exist. The corresponding spatial wave profiles are calculated using the full nonlinear fluid approach. The results are compared with the STEREO satellite observations.
\end{abstract}

Keywords. Electromagnetics (Plasmas) - Magnetospheric physics (Plasma waves and instabilities) - Space plasma physics (Nonlinear phenomena)

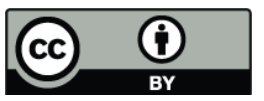

Correspondence to: R. D. Sydora (rsydora@phys.ualberta.ca)

\section{Introduction}

The measurements of large-amplitude whistler waves by the electric field instruments aboard the STEREO satellite in the Earth's radiation belt (Catell et al., 2008) and THEMIS (Cully et al., 2008) has stimulated the investigation of mechanisms by which large amplitude obliquely propagating whistler waves can be generated. Electron temperature anisotropy with $T_{\mathrm{e} \perp}>T_{\mathrm{e} \|}$ can be excluded as a free energy source since the growth rate always has its maximum for parallel propagation. Nevertheless, the simultaneous observation of parallel and obliquely propagating whistler waves has already been described in earlier papers on chorus emission (e.g. Goldstein and Tsurutani, 1984). In the present paper, another possible source of free energy is considered consisting of electron beams which may couple to whistler waves if an electrostatic component arises at oblique wave propagation. For instability analysis, fluid and kinetic models have been developed. The main results are the following; depending on the beam velocity $\left(V_{\mathrm{b}}\right)$ with respect to the Alfvén velocity $\left(V_{\mathrm{Ae}}=B_{\mathrm{o}} /\left(\mu_{\mathrm{o}} n_{\mathrm{po}} m_{\mathrm{e}}\right)^{1 / 2}\right)$, two different mechanisms of beam-plasma interaction may occur. In the regime of $V_{\mathrm{b}}<0.5 V_{\mathrm{Ae}}$, interaction of the beam mode $\omega \sim k V_{\mathrm{b}}$ with the whistler wave takes place (Cerenkov instability). For higher beam velocities $V_{\mathrm{b}}>1.5 V_{\mathrm{Ae}}$ the instability is caused by the Doppler-shifted mode $\omega=-\Omega_{\mathrm{e}}+$ $k V_{\mathrm{b}} \cos \theta$ (cyclotron-type instability, $\Omega_{\mathrm{e}}=e B_{\mathrm{o}} / m_{\mathrm{e}}$ ) and has its maximum growth rate at $k c / \omega_{\mathrm{e}} \sim 1$. With increasing beam velocity $V_{\mathrm{b}}$, the propagation angle which belongs to the maximum increment shifts from nearly zero, at about $V_{\mathrm{b}} \sim 1.6 V_{\mathrm{Ae}}$, up to values close to 90 degrees if $V_{\mathrm{b}} / V_{\mathrm{Ae}}$ becomes larger than about 3-4. Modifications of the electromagnetic fluid dispersion theory due to finite temperature effects (Vlasov kinetic theory) are small as long as the electron plasma beta, the ratio between electron thermal and magnetic pressure defined by $\beta_{\mathrm{e}}=n_{\mathrm{eo}} k T_{\mathrm{e}} /\left(B_{\mathrm{o}}^{2} / 2 \mu_{\mathrm{o}}\right)$, is below $\beta_{\mathrm{e}} \sim 0.2$.

Published by Copernicus Publications on behalf of the European Geosciences Union. 


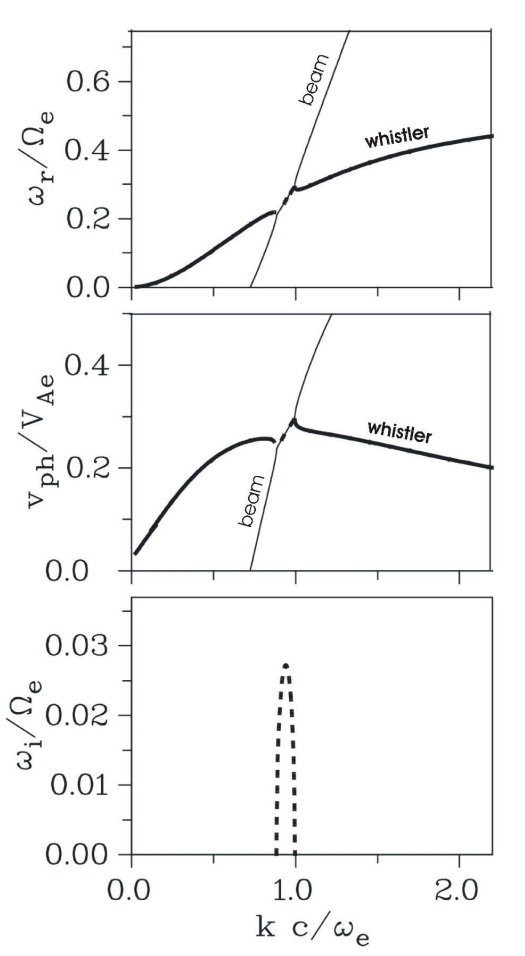

Fig. 1. Fluid dispersion of beam-excited whistlers at oblique propagation $\left(\theta=60^{\circ}\right)$; the beam density is $n_{\mathrm{b}}=0.01 n_{\mathrm{po}}$, the beam velocity is $V_{\mathrm{b}}=2.5 V_{\mathrm{Ae}}$. From top to bottom: real part of $\omega$ normalized to $\Omega_{\mathrm{e}}$, phase velocity normalized to $V_{\mathrm{Ae}}$ and imaginary part of $\omega$ in normalized units versus the normalized wave number $k c / \omega_{e}\left(\omega_{\mathrm{e}}\right.$ is the electron plasma frequency). The thick solid lines in the upper two panels represent the whistler mode, the thin lines mark the Doppler-shifted cyclotron mode $\omega=-\Omega_{\mathrm{e}}+k V_{\mathrm{b}} \cos (\theta)$. The instability occurs where both modes intersect.

The paper is organized as follows. In Sect. 2 the main steps are described in the derivation of the dispersion relation of beam-excited whistlers using a fluid approach. The resultant polynomial of sixth order $\omega=\omega(k)$ easily allows for the analysis of instability by calculating the growth rate as a function of the main parameters such as beam density, beam velocity and propagation angle. Gendrin mode waves $\left(k c / \omega_{e} \approx 1\right)$ are of special relevance since analytical relations for optimum conditions can be obtained. Vlasov dispersion theory is used as well in order to determine the influence of finite electron temperature. It is shown that the instability disappears if the electron plasma beta $\left(\beta_{\mathrm{e}}\right)$ exceeds a value of about 0.2. In Sect. 3 the theory of stationary nonlinear whistlers (whistler oscillitons) is applied to the obliquely propagating modes. Linear theory in the moving frame is used to predict the region of periodic and stationary waves. To compute the spatial profiles of whistler oscillitons, a formalism based on the two fluid approach is presented along with inter-relationships between various electric field components that can be used as a test for the pres-

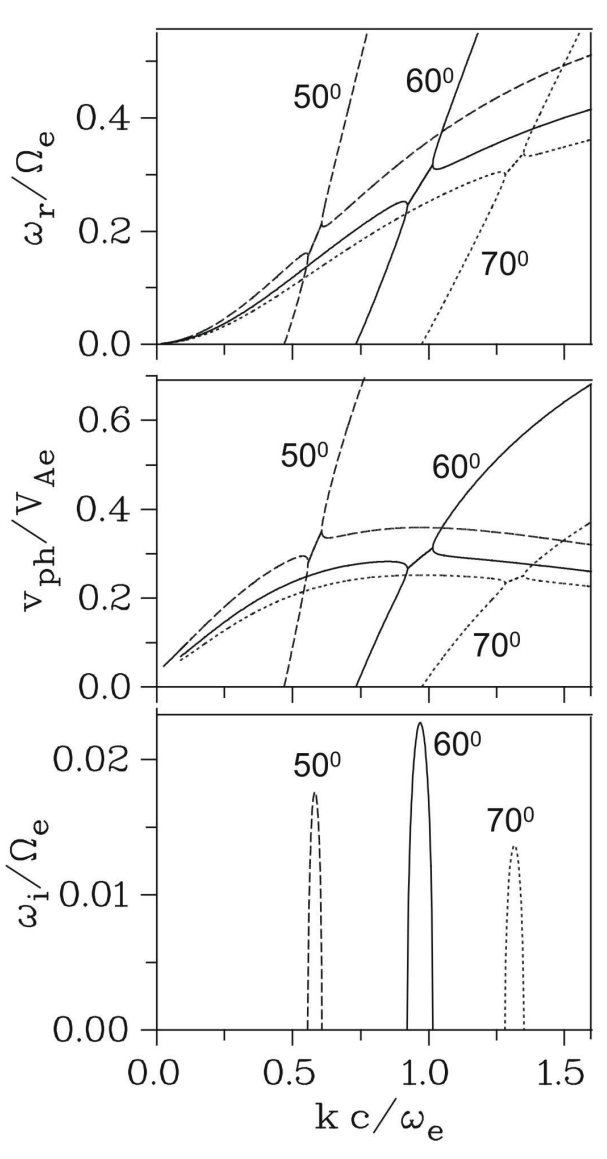

Fig. 2. The same as in Fig. 1, but for different propagation angles. The growth rate has a maximum at $\theta=60^{\circ}$ which appears at $k c / \omega_{\mathrm{e}} \approx 1$.

ence of these nonlinear structures. Finally, in Sect. 4 the results are discussed in relation to the recent observations of large-amplitude waves made by the STEREO satellite in the Earth's radiation belt.

\section{Dispersion of beam-excited whistlers}

\subsection{Fluid approach}

To derive the dispersion relation of beam-excited whistlers, first a simple plasma model is used assuming that all plasma populations; protons, main and beam electrons, are cold. Protons are taken into account in order to prevent the limitations for wave propagation near $90^{\circ}$ (lower-hybrid frequency range). The beam propagates parallel to the ambient magnetic field. The cold plasma model is a good approximation as long as the electron cyclotron damping of whistlers is negligible, which is the case (as shown later) when the electron temperature is small enough. Expressed by the electron plasma beta $\left(\beta_{\mathrm{e}}\right)$, this requires a value of $\beta_{\mathrm{e}} \leq 0.2$. Compared 
with the kinetic description (Vlasov approach in Sect. 2.2), the fluid model has the advantage that the dispersion relation $\omega(k)$ can be expressed in an algebraic form as a polynomial which easily allows analysis of the parameter dependence of the unstable solutions.

As a starting point, the fluid equations of protons, main and beam electrons (marked by $p, e$ and $b$, respectively) are solved together with the Maxwell equations. The procedure to derive the dispersion relation follows the common steps. After linearization and Fourier transform, the fluid equations can be written in the following form

$\mathbf{L}_{\mathrm{p}} \cdot \mathbf{v}_{\mathrm{p}}=c_{p} \boldsymbol{E}, \quad \mathbf{L}_{\mathrm{e}} \cdot \mathbf{v}_{\mathrm{e}}=c_{e} \boldsymbol{E}, \quad \mathbf{L}_{\mathrm{b}} \cdot \mathbf{v}_{\mathrm{b}}=\mathbf{W}_{\mathrm{b}} \cdot \boldsymbol{E}$

$\mathbf{F} \cdot \boldsymbol{E}=c_{j}\left(-n_{\mathrm{eo}} \mathbf{v}_{\mathrm{e}}-n_{\mathrm{bo}} \mathbf{N}_{\mathrm{b}} \cdot \mathbf{v}_{\mathrm{b}}+n_{\mathrm{po}} \mathbf{v}_{\mathrm{p}}\right)$

where $n_{\mathrm{po}}, n_{\mathrm{eo}}$ and $n_{\mathrm{bo}}$ are the background densities of protons, main and beam electrons, which, under the assumption of quasi-neutrality, are related as $n_{\mathrm{po}}=n_{\mathrm{eo}}+n_{\mathrm{bo}} \cdot \mathbf{v}_{\mathrm{p}, \mathrm{e}, \mathrm{b}}$ are the corresponding velocity disturbances under the action of the electric field $\boldsymbol{E} . \quad c_{p, e, j}$ are simple constants. $\mathbf{L}_{\mathrm{p}, \mathrm{e}}(\omega, k, \theta), \mathbf{L}_{\mathrm{b}}\left(\omega, k, \omega, V_{\mathrm{b}}\right), \mathbf{W}_{\mathrm{b}}\left(\omega, k, \theta, V_{\mathrm{b}}\right), \mathbf{F}(\omega, k, \theta)$, and $\mathbf{N}_{\mathrm{b}}\left(\omega, k, \theta, V_{\mathrm{b}}\right)$ are square $3 \times 3$ matrices which can easily be calculated and are not given here. $\theta$ is the propagation angle with respect to the ambient magnetic field and $V_{\mathrm{b}}$ is the velocity of the electron beam. Expressing $\mathbf{v}_{\mathrm{p}}, \mathbf{v}_{\mathrm{e}}$ and $\mathbf{v}_{\mathrm{b}}$ from Eq. (1) by means of matrix inversion through the electric field $\boldsymbol{E}$, one gets the relation $\mathbf{M} \cdot \boldsymbol{E}=0$, where the dispersion matrix $\mathbf{M}$ is given by

$$
\begin{aligned}
\mathbf{M}= & \mathbf{F}+c_{j} c_{e} n_{\mathrm{eo}} \mathbf{L}_{\mathrm{e}}^{-1}+c_{j} c_{e} n_{b o} \mathbf{N}_{b} \cdot \mathbf{L}_{\mathrm{b}}^{-1} \cdot \mathbf{W}_{\mathrm{b}} \\
& -c_{j} c_{p} n_{\mathrm{po}} \mathbf{L}_{\mathrm{p}}^{-1}
\end{aligned}
$$

Finally, the dispersion relation $\omega=\omega(k)$ follows from $D(\omega, k)=\operatorname{Det}(\mathbf{M})=0$ which represents a polynomial of sixth order in $\omega$ and can be solved numerically by standard routines.

In the following, only super-Alfvénic beams, that means $V_{\mathrm{b}}>V_{\mathrm{Ae}}$, are considered. Then, the instability arises from the interaction of the Doppler-shifted cyclotron mode $(\omega=$ $\left.-\Omega_{\mathrm{e}}+k V_{\mathrm{b}} \cos \theta\right)$ with the whistler mode. This is clearly seen in Fig. 1, showing the dispersion relation for the case of a beam with $n_{\mathrm{b}} / n_{\mathrm{po}}=0.01, V_{\mathrm{b}}=2.5 V_{\mathrm{Ae}}$ and propagation angle $\theta=60^{\circ}$ is taken. The thin straight line in the upper panel of Fig. 1 represents the "beam mode" (marked by $b$ ), and the plasma becomes unstable where this mode intersects the whistler mode (thick line). How the growth rate and the related wave number vary with the propagation angle $\theta$ is shown in Fig. 2 where (using the same beam parameters as in Fig. 1) the dispersion curves are plotted for $\theta=50^{\circ}, 60^{\circ}$, and $70^{\circ}$. Clearly, the maximum growth rate is reached near $\theta=60^{\circ}$ and the corresponding wave number is at $k c / \omega_{\mathrm{e}} \sim 1$. This is a more general signature as seen in the next figure. In Fig. 3 the growth rate of beam-excited whistlers is plotted as a function of the propagation angle taking different beam velocities $\left(V_{\mathrm{b}} / V_{\mathrm{Ae}}=2,3,4\right)$ as the parameters. The beam

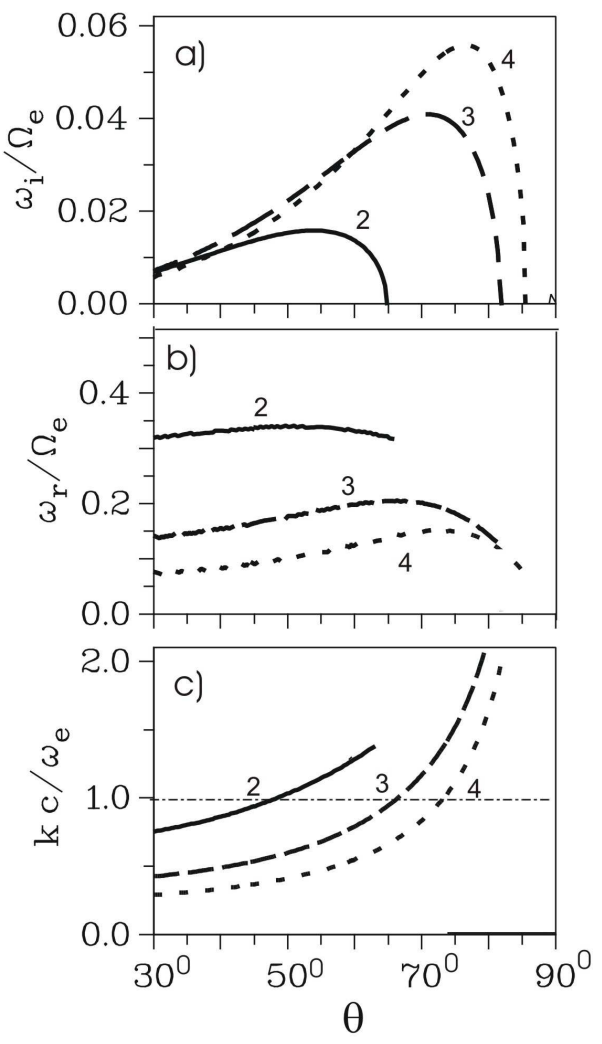

Fig. 3. From top to bottom: (a) Maximum growth rate, (b) related (real) frequency and (c) wave number versus the propagation angle $\theta$, for different beam velocities (in units of $V_{\mathrm{Ae}}$ ). The beam density is $n_{\mathrm{b}} / n_{\mathrm{po}}=0.01$. Note that the wave number for maximum growth rate is at $k c / \omega_{\mathrm{e}} \approx 1$.

density is fixed at $n_{\mathrm{b}} / n_{\mathrm{po}}=0.01$. A significant feature is that the optimum propagation angle shifts closer to $90^{\circ}$ if the beam velocity increases. Further, changing the beam velocity from $V_{\mathrm{b}}=2 V_{\mathrm{Ae}}$ to $V_{\mathrm{b}}=4 V_{\mathrm{Ae}}$ is accompanied by a decrease of the related frequency from about $\omega / \Omega_{e} \sim 0.35$ to 0.15 . In all cases, however, the maximum growth rate remains at $k c / \omega_{\mathrm{e}} \sim 1$ which is just the point where the phase velocity of the whistler mode has its maximum and coincides with the group velocity. As discussed in an earlier paper by Sauer et al. (2002) the coincidence of phase and group velocity is a necessary condition for the existence of the specific class of stationary nonlinear whistlers ("whistler oscillitons"). This is a topic of interest on it's own that will be discussed separately later (see Sect. 3). Finally, Fig. 4 summarizes how the optimum growth rate, the related frequency and propagation angle, vary as a function of the beam velocity. Once again, based on this analysis, one can conclude that the observation of strongly inclined whistlers $\left(\theta \geq 60^{\circ}\right)$ may indicate the presence of super-Alfvénic electron beams with $V_{\mathrm{b}} \geq 2 V_{\mathrm{Ae}}$.

Our numerical studies of beam-excited whistlers are completed by the addition of some useful analytical expressions 


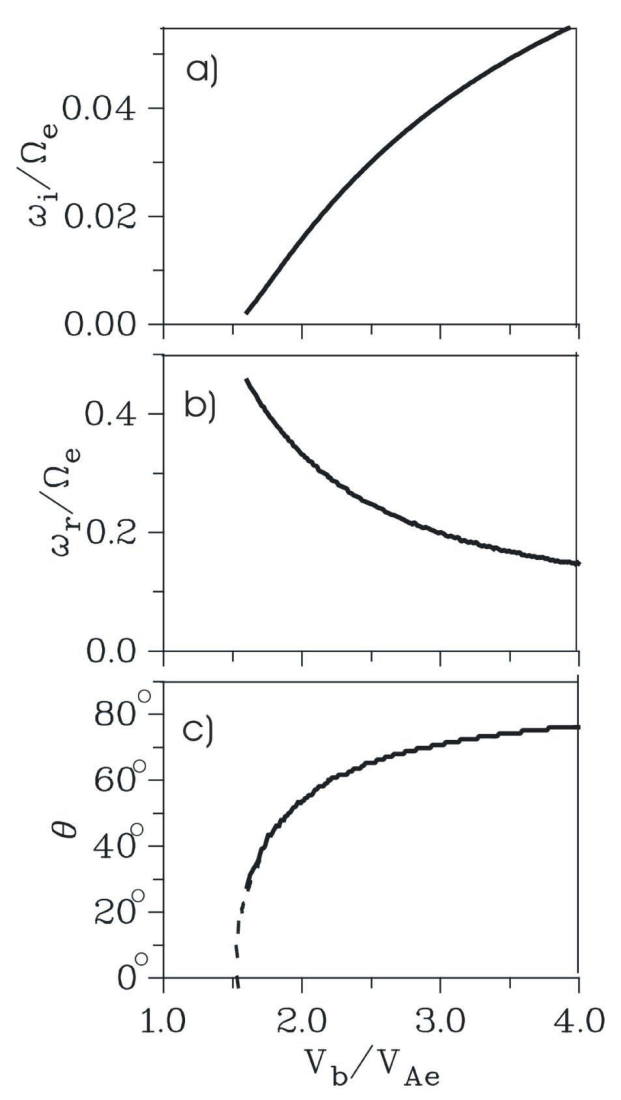

Fig. 4. (a) Optimum growth rate, (b) related (real) frequency and (c) propagation angle $\theta$ as a function of the beam velocity $V_{\mathrm{b}} / V_{\mathrm{Ae}}$; $n_{\mathrm{b}} / n_{\mathrm{o}}=0.01$. With higher beam velocity the growth rate increases and the propagation angle $\theta$ shifts closer to $90^{\circ}$. The relations (b) and (c) can be expressed analytically by Eqs. (5) and (7), respectively.

that are related to the specific whistler wave mode with $k c / \omega_{e}=1$, which has been called the Gendrin mode (Gendrin, 1961; Verkhoglyadova and Tsurutani, 2009). If we start from the whistler dispersion relation, assuming $\omega_{e} \gg \Omega_{e}$, the real frequency is expressed as

$$
\frac{\omega}{\Omega_{\mathrm{e}}}=\frac{\cos \theta}{1+\frac{\omega_{\mathrm{e}}^{2}}{k^{2} c^{2}}}
$$

and the phase velocity $\left(v_{\mathrm{ph}}\right)$ and propagation angle $(\theta)$ at $k c / \omega_{\mathrm{e}}=1$ (Gendrin point) are related through

$$
\frac{\omega}{\Omega_{\mathrm{e}}}=\frac{v_{\mathrm{ph}}}{V_{\mathrm{Ae}}}=\frac{\cos \theta}{2}
$$

Furthermore, the phase velocity has a maximum for different $k$ values and is equal to the group velocity (Sauer et al., 2002; Dubinin et al., 2003). Based on the numerical studies presented in Figs. 1-3, the growth rate of the beam-excited whistlers maximizes at $k c / \omega_{\mathrm{e}} \simeq 1$ from the intersection of the Gendrin mode $\left(\omega / \Omega_{\mathrm{e}}=0.5 \cos \theta\right)$ and the beam mode

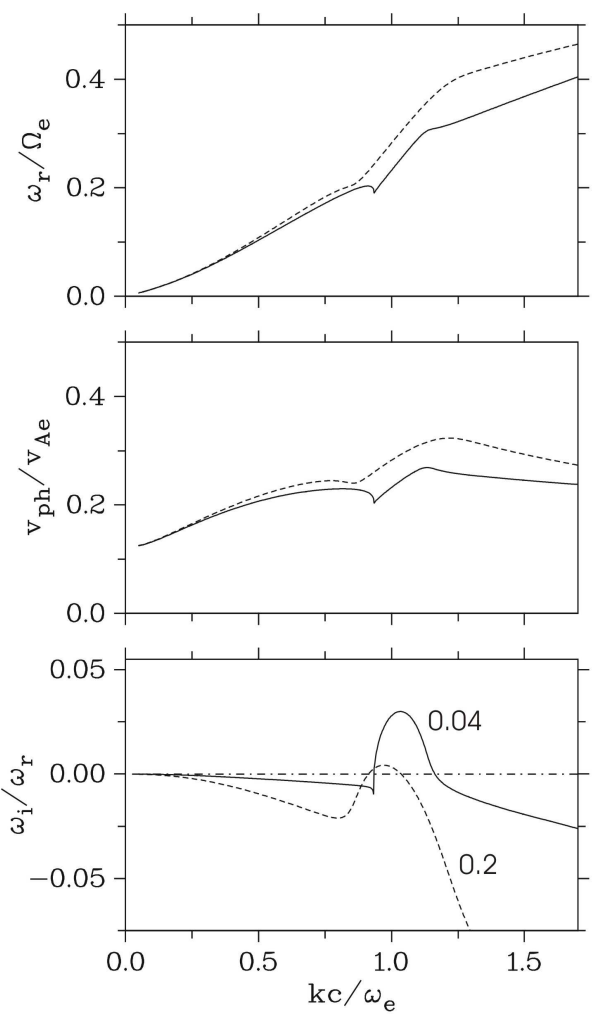

Fig. 5. Vlasov dispersion of beam-excited whistlers at oblique propagation $\left(\theta=70^{\circ}\right)$ for two values of electron plasma beta $\left(\beta_{\mathrm{e}}=\right.$ 0.04-solid lines, $\beta_{\mathrm{e}}=0.2$-dashed lines), showing the effect of kinetic damping on the growth rate. The other parameters are: $n_{\mathrm{b}} / n_{\mathrm{po}}=0.05, V_{\mathrm{b}} / V_{\mathrm{Ae}}=3.0$.

$\left(\omega / \Omega_{\mathrm{e}}=-1+\left(V_{\mathrm{b}} / V_{\mathrm{Ae}}\right) \cos \theta\right)$. Therefore, one finds a relation between the beam velocity and the (optimum) propagation angle as

$\frac{V_{\mathrm{b}}}{V_{\mathrm{Ae}}} \simeq \frac{1}{2}+\frac{1}{\cos \theta}$

or

$\cos \theta \simeq\left(\frac{V_{\mathrm{b}}}{V_{\mathrm{Ae}}}-\frac{1}{2}\right)^{-1}$.

For instance, if we take $V_{\mathrm{b}} / V_{\mathrm{Ae}}=4$, one obtains a propagation angle of $\theta=73.4^{\circ}$, which is in full agreement with the value obtained in Fig. 4. Furthermore, Eq. (6) immediately gives the minimum beam speed of $V_{\mathrm{b}} / V_{\mathrm{Ae}}=1.5$, a value also obtained from the same figure.

\subsection{Kinetic approach}

In the kinetic approach the full apparatus of Vlasov dispersion theory is used, expressing the matrix elements of the susceptibility tensor by Bessel functions $I_{n}(\lambda)$ and the plasma dispersion function $W(z)$ as described, e.g., in the book by Stix (1992). To study beam-excited whistlers the 
distribution functions of protons and main electrons are expressed by Maxwellians with given temperatures $T_{\mathrm{p}}$ and $T_{\mathrm{e}}$; for the beam, a shifted Maxwellian is taken, characterized by the beam velocity $V_{\mathrm{b}}$ and the beam temperature $T_{\mathrm{b}}$. The kinetic dispersion relation, which is a transcendental equation for $\omega=\omega(k, \theta)$ with complex $\omega$ and real $k$, is solved by a Newton root finding method. The knowledge of solutions using the cold plasma fluid approach of the previous section has been proven to be very helpful in finding the corresponding kinetic solutions in the case of finite temperatures. Figure 5 shows the results of solving the kinetic dispersion relation comparable to that of Fig. 1 indicating the effect of kinetic damping on the growth rate. The essential parameter is the electron plasma beta and as long as $\beta_{\mathrm{e}}$ is smaller than about 0.05 , both the kinetic and fluid approaches give nearly the same results. The instability is suppressed if $\beta_{\mathrm{e}}$ exceeds about 0.2 . These values weakly change with the beam density which was taken as $n_{\mathrm{b}} / n_{\mathrm{po}}=0.01$ in Fig. 1 .

The formalism of dispersion theory is also applied to determine the polarization of whistler waves at oblique propagation and to see how it is affected by thermal effects. For later comparison with space measurements it is necessary to perform a linear coordinate transformation $(x, y, z) \rightarrow$ $\left(x^{\prime}, y^{\prime}, z^{\prime}\right)$ : the original coordinate frame with the undisturbed magnetic field in the $\mathrm{z}$-direction is rotated through the angle $\theta$ about the $y$-axis in such a way that the new $\mathrm{z}^{\prime}$-axis is aligned in the direction of wave propagation $k$. In the lower two panels of Fig. 6, the polarization in the $\mathrm{z}^{\prime}-\mathrm{x}^{\prime}$ and $\mathrm{x}^{\prime}-\mathrm{y}^{\prime}$ planes $\left(E_{z}^{\prime} / E_{x}^{\prime}, E_{x}^{\prime} / E_{y}^{\prime}\right)$ is plotted versus $k c / \omega_{\mathrm{e}}$, taking $\theta=70^{\circ}$ and $\beta_{\mathrm{e}}=0.2$. The corresponding whistler dispersion (real and imaginary parts of the frequency) is shown in the top panel. For comparison, the outcome of the cold plasma theory is drawn as thin lines. The straight dashed line in the middle panel $E_{x}^{\prime} / E_{y}^{\prime}=i$, which belongs to a cold plasma, means that the whistler waves are right-hand circularly polarized in a plane normal to the wave propagation direction. That is generally valid, independent of the propagation angle and wave number. The situation becomes more complicated for finite electron plasma beta $\left(\beta_{\mathrm{e}}\right)$ as indicated by the appearance of the complex amplitude ratio (thick solid and dashed lines). The polarization in the $\mathrm{x}^{\prime}-\mathrm{z}^{\prime}$ plane, on the other hand, is linear in the cold plasma approximation (thin solid line in the bottom panel); it varies from $E_{z}^{\prime} / E_{x}^{\prime}=\tan \theta$ at $k=0$ to $E_{z}^{\prime} / E_{x}^{\prime}=2 \tan \theta$ at the Gendrin point $k c / \omega_{\mathrm{e}}=1$ indicating the existence of a dominant longitudinal electric field component at highly oblique propagation. With increasing electron temperatures, the ratio $E_{z}^{\prime} / E_{x}^{\prime}$ becomes increasingly modified due to the fact that real and imaginary parts become comparable.

\section{Whistler oscillitons (Nonlinear Gendrin modes)}

Whistler oscillitons are stationary nonlinear structures, like solitons but superimposed by spatial oscillations, as first de-

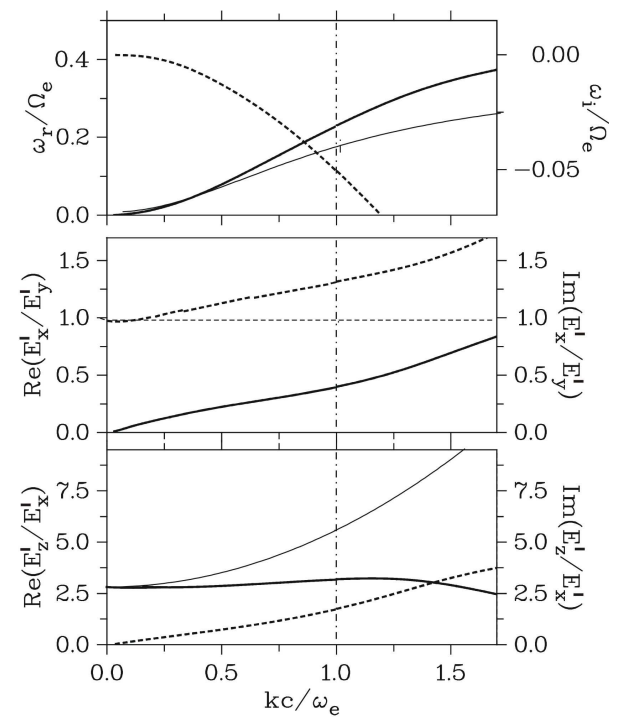

Fig. 6. Dispersion and polarization of whistler waves: $\theta=70^{\circ}$, $\beta_{\mathrm{e}}=0.2$. From top to bottom: frequency and amplitude ratios $\left(E_{z}^{\prime} / E_{x}^{\prime}\right)$ and $\left(E_{x}^{\prime} / E_{y}^{\prime}\right)$ representing the polarization in the $\mathrm{z}^{\prime}-\mathrm{x}^{\prime}$ and $\mathrm{x}^{\prime}-\mathrm{z}^{\prime}$ plane, respectively. Note, that the polarization has been calculated in a coordinate system in which the $\boldsymbol{k}$-vector is directed along the (new) $z^{\prime}$-axis, which is rotated by the angle $\theta$ with respect to the magnetic field direction. Solid (thick) lines mark the real parts, dashed lines the imaginary parts of the three complex quantities. The thin (solid and dashed) lines denote the outcome of cold plasma theory.

scribed in papers by Sauer et al. (2002) and Dubinin et al. (2003). A necessary condition for the existence of oscillitons is a particular dispersion behavior of the underlying wave mode in such a way that the dispersion curve $\omega=\omega(k)$ contains a point in which phase and group velocity coincide. For whistlers, that is the case at $k c / \omega_{e}=1$, independent of the propagation angle. As already mentioned in Sect. 2.1, the corresponding waves are also called Gendrin modes (Gendrin, 1961). Their physical relevance has recently been discussed in a paper by Verkhoglyadova and Tsurutani (2009). Several indications brought us to the conclusion that the nonlinear saturation of beam-excited whistlers at oblique propagation may be directly associated with the formation of whistler oscillitons (a kind of nonlinear Gendrin mode wave). One hint came from our earlier studies of the nonlinear behavior of parallel propagating whistlers which are excited by another type of instability, namely by a temperature anisotropy (Sydora et al., 2007). Using PIC simulations we have shown that the anisotropy instability saturation leads to quasi-stationary structures which have signatures of whistler oscillitons. An interesting effect of the formation of oscilltons concerns the observed wave number shift. If the instability has a maximum growth at wave numbers $k c / \omega_{\mathrm{e}}>1$, for example, a shift to $k c / \omega_{\mathrm{e}} \sim 1$ takes place and, finally, the 

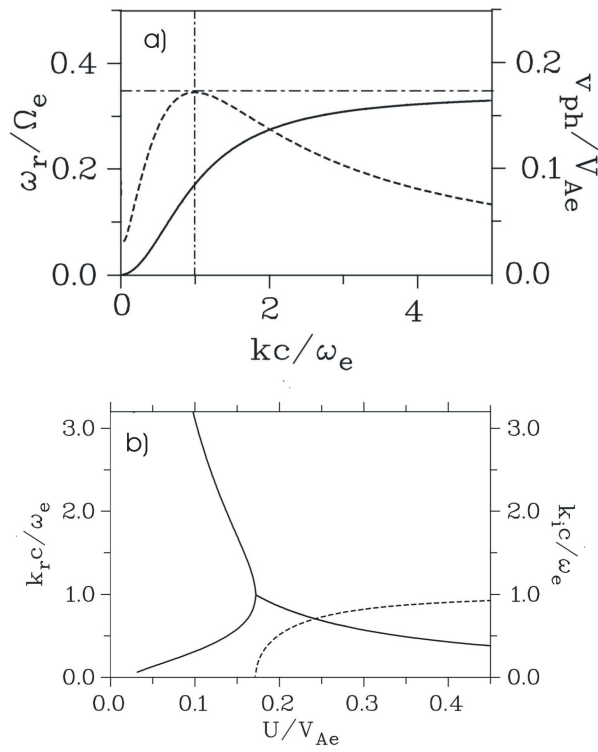

Fig. 7. (a) Dispersion relation of whistler waves at oblique propagation $\left(\theta=70^{\circ}\right)$ : normalized frequency (solid line) and phase velocity (dashed line) versus wave number $k$ (in units of the electron skin length $\left.c / \omega_{\mathrm{e}}\right)$. Maximum phase velocity is at $k c / \omega_{\mathrm{e}}=1$. b) Dispersion relation of stationary whistlers: $k=k(U) ; k_{r}$ (solid line), $k_{i}$ (dashed line) are the real and imaginary parts of $k$, respectively, where $U$ is the velocity of the moving frame. Exponentially growing solutions $\left(k_{i} \neq 0\right)$ superimposed by spatial oscillations $\left(k_{r} \neq 0\right)$ exists for $U / V_{\mathrm{Ae}} \geq 0.17$. That is the region where whistler oscillitons are expected.

resulting waveform of the quasi-stationary state can well be fitted by spatial profiles of whistler oscillitons. We anticipate a similar situation for beam-excited whistlers where the optimum growth rate is already close to $k c / \omega_{\mathrm{e}}=1$, independent of the beam velocity; see Fig. 3 .

We begin with a few simple results from linear dispersion theory which allows for the determination of conditions under which whistler oscillitons may exist. In Fig. 7a the frequency (solid line) and phase velocity (dashed line) of whistlers are plotted versus $k c / \omega_{\mathrm{e}}$ for a propagation angle of $\theta=70^{\circ}$. As can be seen, the phase velocity $\left(v_{\mathrm{ph}}\right)$ has a maximum at $k c / \omega_{\mathrm{e}}=1$ and reaches $v_{\mathrm{ph}} / V_{\mathrm{Ae}} \sim 0.17$ at that wavenumber. Periodic waves with higher phase velocities do not exist. But this is just the region of stationary waves which have no time dependence in a frame that moves with a velocity $U$ larger than the maximum phase velocity, that is $U \geq 0.17 V_{\mathrm{Ae}}$. For the transition to a moving frame, in the dispersion relation $\omega=\omega(k)$ one has to replace $\omega$ by $\omega=\omega^{\prime}+k U$ (with $\omega^{\prime}=0$ for stationary waves), and a relation for $k=k(U)$ is obtained, which is plotted in Fig. 7b. In the region $U \geq 0.17 V_{\mathrm{Ae}}$ the wave number $k$ is complex which means growing solutions with the increment $k_{\mathrm{i}}$ are superimposed by spatial oscillations whose wavelength $\lambda$ is determined by $\lambda=2 \pi / k_{r}$. It should be noted that near the border between periodic and stationary waves the wave number $k_{r}$ is always given by $k_{r} c / \omega_{e} \sim 1$.

The next step is to calculate the spatial profiles of whistler oscillitons. To derive the governing equations which describe stationary nonlinear whistlers, one starts from the fluid equations of electrons and protons together with the Maxwell equations. Opposite to the assumptions in earlier papers (Sauer et al., 2002; Dubinin et al., 2003), the analysis is carried out in the plasma rest frame in which the structure moves with the velocity $U$ in $\mathrm{x}$-direction. The undisturbed magnetic field lies in the $\mathrm{x}-\mathrm{z}$ plane and is inclined by the angle $\theta$ relative to the x-axis, $\boldsymbol{B}=B_{0}(\cos \theta, 0, \sin \theta)$. Looking for stationary waves means finding solutions of the Maxwell-fluid equations in which the time dependence appears only in the form $f(x-U t)$. Since the more general formalism has been described in earlier papers, only a summary of equations is given which we used for our calculations and which we need for later discussion.

The transverse velocities (velocity components transverse to the propagation direction $x$ ) of both species are calculated by means of ordinary differential equations which directly follow from the basic fluid equation using the ansatz of stationary solutions $(\partial / \partial t \rightarrow-U d / d x)$

$$
\begin{aligned}
& \frac{d v_{e, p y}}{d x}=\frac{q_{\mathrm{e}, \mathrm{p}}}{\mu_{\mathrm{e}, \mathrm{p}}}\left(E_{y}-v_{e, p x} B_{z}+v_{e, p z} B_{x}\right) /\left(M_{e}-v_{e, p x}\right) \\
& \frac{d v_{e, p z}}{d x}=\frac{q_{\mathrm{e}, \mathrm{p}}}{\mu_{\mathrm{e}, \mathrm{p}}}\left(E_{z}-v_{e, p x} B_{y}+v_{e, p y} B_{x}\right) /\left(M_{e}-v_{e, p x}\right)
\end{aligned}
$$

The velocities are normalized by the (electron) Alfvén velocity based on the electron mass density, $V_{\mathrm{Ae}}=$ $B_{\mathrm{o}} /\left(\mu_{\mathrm{o}} n_{\mathrm{eo}} m_{\mathrm{e}}\right)^{1 / 2}$. The electric field is given in units of $E_{\mathrm{o}}=$ $V_{\mathrm{Ae}} B_{\mathrm{o}}$, the magnetic field is normalized by $B_{\mathrm{o}} . M_{\mathrm{e}}=U / V_{\mathrm{Ae}}$ is the electron Mach number of the moving structure at infinity. The other quantities are the electric charge and the mass of electrons and protons in units of the electron mass, respectively: $q_{\mathrm{e}}=-1, q_{\mathrm{p}}=+1, \mu_{\mathrm{e}}=1, \mu_{\mathrm{p}}=m_{\mathrm{p}} / m_{\mathrm{e}}$.

From Faraday's law one gets

$E_{y}=M_{\mathrm{e}}\left(B_{z}-B_{z o}\right), \quad E_{z}=-M_{\mathrm{e}} B_{y}$

The longitudinal electric field component $E_{x}$ can be determined from the transverse field condition $\boldsymbol{E} \times \boldsymbol{B}=0$, that is

$$
\begin{aligned}
E_{x} & =-\left(E_{y} B_{y}+E_{z} B_{z}\right) / B_{x}=-M_{e} \tan \theta B_{y} \\
& =\tan \theta E_{z}
\end{aligned}
$$

Conservation of mass and (longitudinal and transverse) momentum together with the quasi-neutrality condition $n_{\mathrm{p}}=n_{\mathrm{e}}$, combined with zero current in $\mathrm{x}$-direction, $j_{x}=0$, which yields $v_{e x} \approx v_{p x}$, one gets the following equations for the remaining quantities $v_{p x}, B_{y}, B_{z}$ as follows

$$
\begin{aligned}
v_{p x} & \approx v_{e x}=\frac{1}{2 M_{\mathrm{e}} \mu_{p}}\left(B^{2}-1\right) \\
B_{y} & =-M_{\mathrm{e}}\left(\mu_{\mathrm{p}} v_{p y}+\mu_{\mathrm{e}} v_{e y}\right) / B_{x} \\
B_{z} & =-M_{\mathrm{e}}\left(\mu_{\mathrm{p}} v_{p z}+\mu_{\mathrm{e}} v_{e z}\right) / B_{x}
\end{aligned}
$$




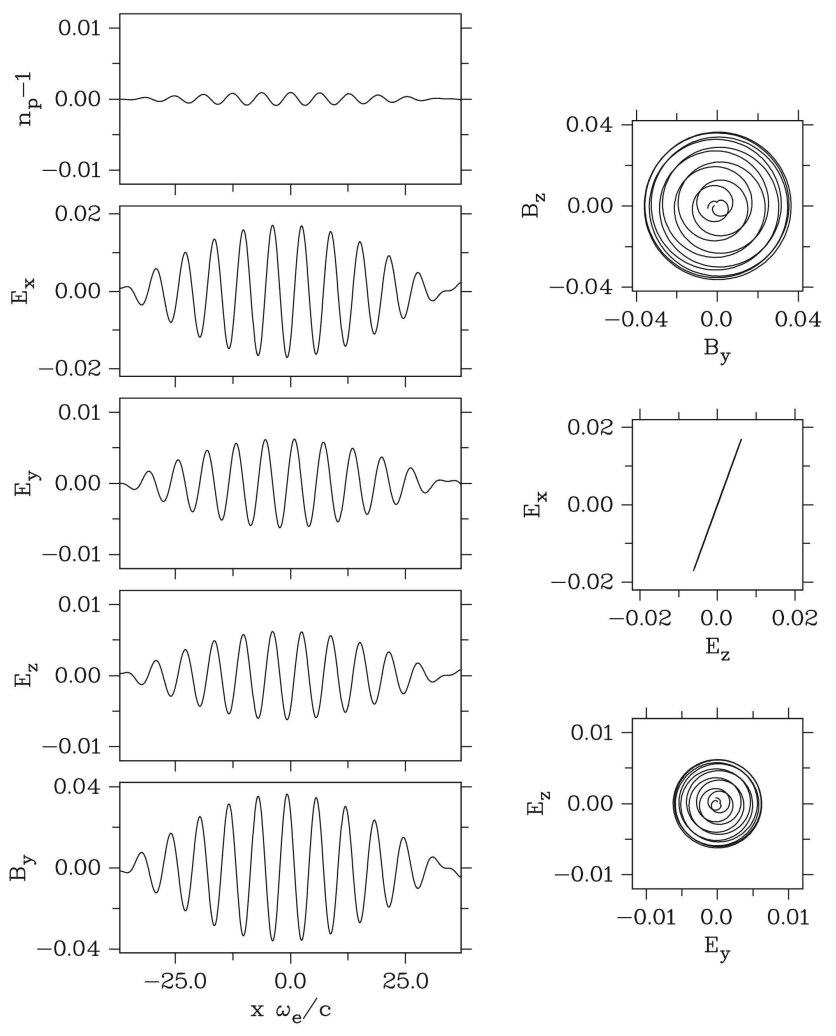

Fig. 8. Spatial profiles of whistler oscillitons for $\theta=70^{\circ}$ and $U=$ $0.172 V_{\mathrm{Ae}}$. From top to bottom: proton density $\left(n_{\mathrm{p}}-n_{\mathrm{po}}\right) / n_{\mathrm{po}}$, three components of the electric field (in units of $E_{\mathrm{O}}=V_{\mathrm{Ae}} B_{\mathrm{O}}$ ) and the magnetic field component $B_{y} / B_{O}$. The right panels show the hodographs $B_{z}$ versus $B_{y}, E_{x}$ versus $E_{z}$, and $E_{z}$ versus $E_{y}$.

If the propagation angle is chosen, the only free parameter in the system of equations above is the velocity of the moving structure, $M_{\mathrm{e}}$, also called the oscilliton speed. For a propagation angle of $\theta=70^{\circ}$, taken from Fig. 7 of the previous section, whistler oscillitons should exist for $M_{\mathrm{e}} \geq 0.17$. In Fig. 8 the corresponding spatial profiles of the electric and magnetic field components are plotted using $M_{\mathrm{e}}=0.172$. They clearly exhibit a soliton-like structure with superimposed oscillations representing the expected nonlinear configuration in the form of a whistler oscilliton.

One has to take in to consideration that the oscilliton profile (amplitude and extension of the nonlinear wave packet) varies if the oscilliton speed $M_{\mathrm{e}}$ changes. This is depicted in Fig. 9 where the spatial profile of the longitudinal electric field amplitude $E_{x} / E_{\mathrm{o}}$ is plotted for three values of $M_{\mathrm{e}}$ which are all slightly above $M_{\mathrm{e}}=0.17$, but close together $\left(M_{\mathrm{e}}=0.1718,0.1721,0.1723\right)$. As one would expect from Fig. $7 \mathrm{~b}$, the wavelength always remains the same and is given by $k_{r} c / \omega_{\mathrm{e}} \sim 1$. In comparison, the oscilliton waveform (amplitude and spatial extent) changes considerably. An increase of the amplitude is accompanied by a larger

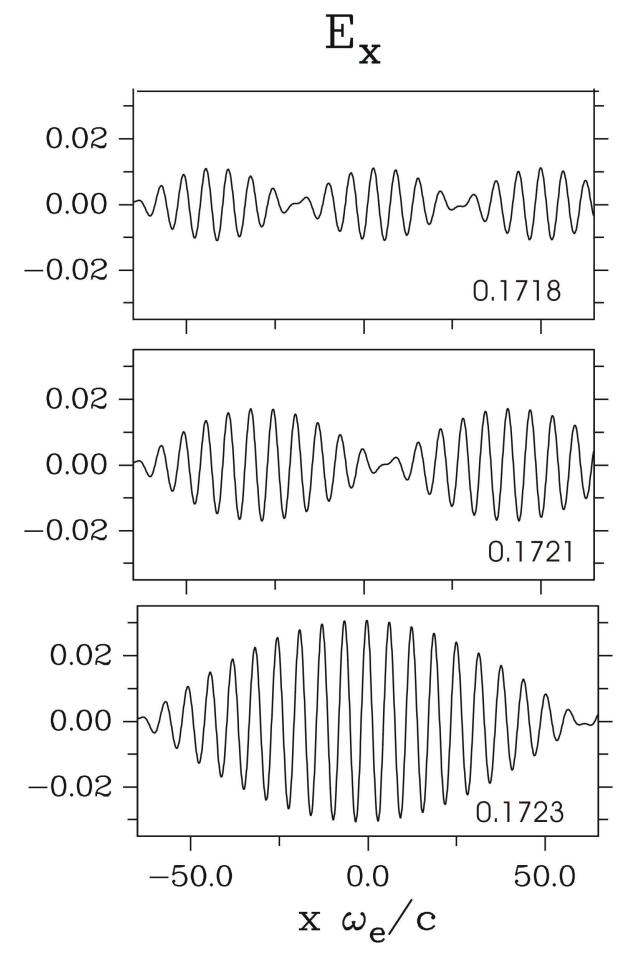

Fig. 9. Spatial profiles of the longitudinal electric field component (in units of $E_{\mathrm{O}}=V_{\mathrm{Ae}} B_{\mathrm{O}}$ ) for three values of the oscilliton speed $M_{\mathrm{e}}=U / V_{\mathrm{Ae}}$. Obviously the waveform is very sensitive to values of $M_{\mathrm{e}}$. For $M_{\mathrm{e}} \geq 0.1725$ whistler oscillitons no longer exist.

wavepacket length and the latter changes from about 7 to 20 wavelengths. If one selects a waveform with an extension of about 10 wavelengths, for fitting with the observations of Catell et al. (2008), one finds an associated amplitude of $E_{x} / E_{\mathrm{o}} \approx 0.01$. For oscillitons speeds of $M_{\mathrm{e}} \geq 0.1725$ it was not possible to find any more stationary solutions.

Additional information about the wave characteristics can be obtained from the hodograms on the right hand side of Fig. 8. As clearly seen, the wave is circularly polarized in the plane perpendicular to the propagation direction and has a significant electric field component parallel to this direction. The straight line in the middle of the hodogram represents $E_{x}=\tan \theta E_{z}$ according to Eq. (11). A comparable polarization is obtained in the paper by Verkhoglyadova and Tsurutani (2009) in the context of the Gendrin mode analysis.

\section{Summary and discussion}

In this paper, the excitation of whistler waves by an isotropic electron beam parallel to the ambient magnetic field has been studied. Instability occurs for the case of oblique wave propagation due to the interaction of the Doppler-shifted cyclotron mode $\omega=-\Omega_{\mathrm{e}}+k V_{\mathrm{b}} \cos \theta$ with the whistler mode; 

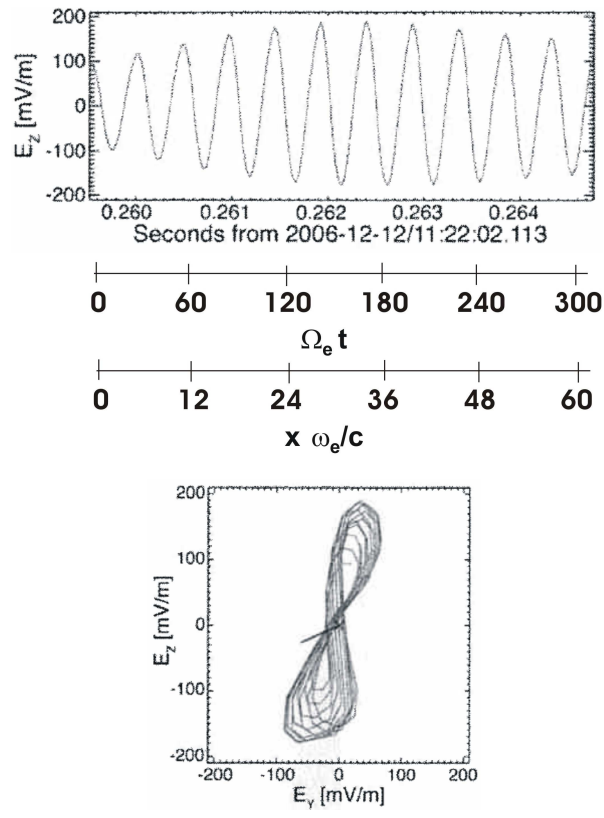

Fig. 10. Measured waveform (top) and hodogram (bottom) of the maximum component versus the intermediate component for the time interval above (adapted from Cattell et al., 2008). The first additional scale is obtained by multiplying the time (from zero to $0.005 \mathrm{~s}$ ) with the electron cyclotron frequency of $\Omega_{\mathrm{e}}=6 \times 10^{4} \mathrm{~s}^{-1}$ according to a magnetic field of $B_{\mathrm{O}} \sim 320 \mathrm{nT}$. The second spatial scale, in units of the electron skin depth $c / \omega_{\mathrm{e}}$, follows directly from the upper one by multiplying it with a normalized phase velocity of $V_{\mathrm{ph}} / V_{\mathrm{Ae}}=0.2$ and allows a direct comparison with the oscilliton waveform plotted in Figs. 8 and 9.

its maximum growth rate is at $k c / \omega_{\mathrm{e}} \sim 1$. In addition, at the particular wave number $k c / \omega_{\mathrm{e}}=1$, the so-called Gendrin mode waves exist. The group velocity of such waves is directed along the magnetic field and coincides with the component of the phase speed parallel to the magnetic field. This, in turn, is a necessary condition for the appearance of stationary nonlinear waves (whistler oscillitons) which, therefore, can be considered as nonlinear Gendrin modes. In this context, the dispersion relation of beam-excited whistler waves in a cold plasma has been analyzed leading to relations between the beam speed and wave propagation characteristics (frequency, growth rate, phase velocity, propagation angle). In addition, the Vlasov approach is applied to check the modifications due to thermal effects. Finally, using a fluid approach, spatial profiles of obliquely propagating whistler oscillitons have been calculated.

Our studies of beam-excited whistler waves have been stimulated by the recent satellite measurements in the Earth's radiation belt. Catell et al. (2008) describe the discovery of large-amplitude whistlers aboard the satellite STEREO-B with peak amplitudes of approximately $240 \mathrm{mV} / \mathrm{m}$. During the passage through the radiation belt, a sequence of wave- form samples have been obtained using the Time Domain Sampler (TDS). As an example, the maximum variance component and the hodogram of that component versus the intermediate component for the same time interval is shown in Fig. 10. A characteristic feature is the occurrence of wave packets with varying shapes and durations. The peak frequency for all TDS measurements was $\omega \sim 0.2 \Omega_{\mathrm{e}}$. A remarkable feature is that the waves propagate very oblique to the geomagnetic field with a propagation angle $(\theta)$ ranging from $45^{\circ}$ to $60^{\circ}$. From the estimated phase velocity $\left(v_{\mathrm{ph}}\right)$ between 35000 and $70000 \mathrm{~km} / \mathrm{s}$ and an electron Alfvén velocity $V_{\mathrm{Ae}}$ of about $135000 \mathrm{~km} / \mathrm{s}$ (using $n_{\mathrm{e}}=4 \mathrm{~cm}^{-3}$ and $B=300 \mathrm{nT}$ ) one obtains a ratio of $V_{\mathrm{ph}} / V_{\mathrm{Ae}} \sim 0.2-0.4$. These values can be used to check whether the conditions for Gendrin mode waves, expressed by Eq. (5), are fulfilled. If we assume a (Gendrin) propagation angle $\theta=60^{\circ}$, then the associated frequency and phase velocity are given by $\omega / \Omega_{\mathrm{e}}=v_{\mathrm{ph}} / V_{\mathrm{Ae}}=$ $\cos \left(60^{\circ}\right) / 2=0.25$, a value relatively close to that of the measurements and estimations, respectively. The required beam velocity, according to Eq. (6), would be $V_{\mathrm{b}} / V_{\mathrm{Ae}} \sim 2.5$ and corresponds to about $0.3 \mathrm{MeV}$. Whether such beams which lie in the range of relativistic velocities $\left(V_{\mathrm{b}}>10^{10} \mathrm{~cm} / \mathrm{s}\right)$ really exist is presently an open question. Another critical problem concerns the electron temperature. With the measured radiation belt density and magnetic field parameters given above, the temperature should not exceed $10 \mathrm{keV}$ to remain in the unstable range of $\beta_{\mathrm{e}} \leq 0.2$. This value is too low for the typical substorm situation and one may ask whether the whistler excitation mechanism considered here requires particular radiation belt conditions.

In this context, the solar wind appears to be another suitable medium to analyze the origin of coherent obliquely propagating whistlers. First examples of simultaneous measurements of large-amplitude whistler wave packets propagating slightly oblique to the magnetic field and beam-like electron distribution functions have been presented by Cattell et al. (2009). Dispersion analysis with $\beta_{\mathrm{e}} \leq 0.5$ shows that instability at small $k\left(k c / \omega_{\mathrm{e}} \leq 0.2\right)$ appears if moderate beam densities $\left(n_{\mathrm{b}} / n_{\mathrm{o}} \sim 0.05\right)$ are taken. It seems that these waves play an important role in understanding the evolution of the solar wind, especially with respect to the strahl and halo properties and heat flux regulation.

Returning to the STEREO radiation belt observations, Fig. 10 shows a measured whistler waveform together with a hodogram adapted from the paper by Catttell et al. (2008). For comparison with the oscilliton waveform plotted in Figs. 8 and 9 , the originally measured temporal variation is transferred to a spatial waveform by assuming that the structure is stationary in a frame moving with the phase velocity. If for the present case a phase (oscilliton) velocity of $v_{\mathrm{ph}} / V_{\mathrm{Ae}}=0.2$ is assumed, the resulting waveform is in good agreement with the spatial profiles of whistler oscillitons (see Figs. 8 and 9). In particular, it means, that the wavelength is specified by $k c / \omega_{\mathrm{e}} \sim 1$, which is clear evidence of Gendrin mode waves and their importance for whistler wave 
emission. This aspect has also been discussed in the paper by Dubinin et al. (2007).

Another interesting signature which one has to note is the observed ratio between the longitudinal and transverse electric field amplitudes (with respect to the wave propagation direction) as depicted in Fig. 10. The hodogram of the maximum variance component versus the intermediate component for the time interval shown above can roughly be approximated by a straight line, $E_{x} \sim 3 E_{z}$. This would indicate linear polarization, close to the theoretical amplitude ratio of Eq. (11); $E_{x}=\tan \theta E_{z}$. To understand what the amplitudes of the electric field components in Fig. $8, E_{i} / E_{0}$, mean in real units $(\mathrm{V} / \mathrm{m})$, reasonable values for $n_{\mathrm{e} 0}$ and $B_{0}$ have to be taken to calculate $E_{0}=V_{\mathrm{Ae}} B_{0}$. Again, we use the parameters of the Earth's radiation belt described in the paper by Catell et al. (2008): $n_{\mathrm{e} 0}=4 \mathrm{~cm}^{-3}$ and $B_{0}=300 \mathrm{nT}$. For these parameters one gets $E_{0}=45 \mathrm{~V} / \mathrm{m}$ and an amplitude of $E_{x} / E_{0}=0.01$ for the longitudinal field. This corresponds to $E_{x} \sim 450 \mathrm{mV} / \mathrm{m}$, which is a value less than twice the measured electric field of $\sim 240 \mathrm{mV} / \mathrm{m}$ and this corresponds well considering the assumptions of the model. Of course, these simple estimations from cold plasma theory are very crude. More detailed polarization analysis using the Vlasov approach has shown that significant modifications arise if kinetic effects become more important with increasing electron temperature.

Finally, we want to point out that a subsequent paper is in preparation in which particle in-cell simulations are used to calculate the quasi-stationary states of beam-excited whistlers. The kinetic PIC simulations (Sydora et al., 2007) make it possible to calculate the whistler waveforms and amplitudes and their dependence on the most relevant parameters of the background plasma and the beam, including thermal effects.

Acknowledgements. This work is supported by a Discovery grant from the Natural Sciences and Engineering Research Council (NSERC) of Canada. One of us (KS) thanks the DFG/DAAD for travel support.

Topical Editor I. A. Daglis thanks S. C. Buchert and another anonymous referee for their help in evaluating this paper.

\section{References}

Cattell, C., Wygant, J. R., Goetz, K., et al.: Discovery of very large amplitude whistler- mode waves in Earth's radiatikon belts, Geophys. Res. Lett., 35, L01105, doi:10.1029/2007GL032009, 2008.

Cattell, C., Breneman, A., Goetz, K., et al.: STEREO observations of large-amplitude, low-frequency waves and their association with corotating interaction regions, Solar Wind 12, Session 2, Saint-Malo, France, 21-26 June 2009.

Cully, C. M., Bonnell, J. W., and Ergun, R. E.: THEMIS observations of long-lived regions of large-amplitude whistler waves in the inner magnetosphere, Geophys. Res. Lett., 35, L17S16, doi:10.1029/2008GL033643, 2008.

Dubinin, E. M., Maksimovic, M., Cornilleau-Wehrlin, N., Fontaine, D., Travnicek, P., Mangeney, A., Alexandrova, O., Sauer, K., Fraenz, M., Dandouras, I., Lucek, E., Fazakerley, A., Balogh, A., and Andre, M.: Coherent whistler emissions in the magnetosphere - Cluster observations, Ann. Geophys., 25, 303-315, doi:10.5194/angeo-25-303-2007, 2007.

Dubinin, E. M., Sauer, K., and McKenzie, J. F.: Nonlinear stationary waves and whistler solitons (oscillitons). Exact solutions, J. Plasma Phys., 69, 305-330, 2003.

Gendrin, R.: Le guidage des whistlers par le champ magnetique, Planet. Space Sci., 5, 274-281, 1961.

Goldstein, B. E. and Tsurutani, B. T.: Wave normal directions of chorus near the equatorial source region, J. Geophys. Res., 89(A5), 2789-2810, 1984.

McKenzie, J. F., Dubinin, E., Sauer, K., and Doyle, T. B.: The application of the constants of motion to nonlinear stationary waves in complex plasmas: a unified fluid dynamic viewpoint, J. Plasma Phys., 70, 431-462, 2004.

Sauer, K., Dubinin, E. M., and McKenzie, J. F.: Wave emission by whistler oscillitons: Application to "coherent lion roars", Geopyhs. Res. Lett., 29, 2226, doi:10.1029/2002GL015771, 2002.

Stix, T. H.: Waves in Plasmas, American Institute of Physics, New York, 1992.

Sydora, R., Sauer, K., and Silin, I.: Coherent whistler waves and oscilliton formation: Kinetic simulations, Geophys. Res. Lett., 34, L22105, doi:10.1029/2007GL031839, 2007.

Verkhoglyadova, O. P. and Tsurutani, B. T.: Polarization properties of Gendrin mode waves observed in the Earth's magnetosphere: observations and theory, Ann. Geophys., 27, 4429-4433, doi:10.5194/angeo-27-4429-2009, 2009. 